\title{
Detection and localization of atypical porcine pestivirus in the testicles of naturally infected, CT-affected piglets
}

\author{
Lilla Dénes ${ }^{1}$, Inés Ruedas-Torres ${ }^{2}$, Anna Szilasi ${ }^{1}$, and Gyula Balka ${ }^{1}$ \\ ${ }^{1}$ Department of Pathology University of Veterinary Medicine 1078 Budapest István Str 2 \\ Hungary \\ ${ }^{2}$ Universidad de Cordoba
}

July 25, 2021

\begin{abstract}
Atypical porcine pestivirus (APPV) belongs to the genus Pestivirus within the family Flaviviridae. Recently, APPV has been identified as the causative agent of congenital tremor (CT) type AII. The disease is a neurological disorder that affects newborn piglets and is characterized by mostly generalized trembling of the animals and often splay legs. CT is well known worldwide, and the virus seems to be highly prevalent in major swine producing areas. However, little is known about the epidemiology of the infection, the transmission and spread of the virus between herds. Here, we show the high prevalence of APPV in processing fluid samples collected from Hungarian pig herds which led us to investigate the cellular targets of the virus in the testicles of newborn piglets affected by CT. By the development of an RNA in situ hybridization assay and the use of immunohistochemistry on consecutive slides, we identified the target cells of APPV in the testicle: interstitial Leydig cells, peritubular myoid cells and endothelial cells of medium-sized arteries. Previous studies have shown that APPV can be found in the semen of sexually mature boars suggesting the role of infected boars and their semen in the transmission of the virus similar to many other members of the Flaviviridae family. As in our case, the virus has not been identified in cells beyond the blood-testis barrier, further studies on infected adult boars' testicles are needed to analyze the possible changes in the cell tropism that enable the virus to be excreted by the semen.
\end{abstract}

\section{Detection and localization of atypical porcine pestivirus in the testicles of naturally infected, CT-affected piglets}

Lilla Dénes ${ }^{1}$, Inés Ruedas-Torres ${ }^{2}$, Anna Szilasi ${ }^{1}$, Gyula Balka ${ }^{1 *}$

${ }^{1}$ Department of Pathology, University of Veterinary Medicine, 1078 Budapest, István Str. 2., Hungary

${ }^{2}$ Department of Anatomy and Comparative Pathology and Toxicology, Faculty of Veterinary Medicine, University of Córdoba, 14014, Córdoba, Spain

*Correspondence:balka.gyula@univet.hu; Tel.: +36-1-478-4181

\section{Summary}

Atypical porcine pestivirus (APPV) belongs to the genusPestivirus within the family Flaviviridae . Recently, APPV has been identified as the causative agent of congenital tremor (CT) type AII. The disease is a neurological disorder that affects newborn piglets and is characterized by mostly generalized trembling of the animals and often splay legs. CT is well known worldwide, and the virus seems to be highly prevalent in major swine producing areas. However, little is known about the epidemiology of the infection, the transmission and spread of the virus between herds. Here, we show the high prevalence of APPV in processing fluid samples collected from Hungarian pig herds which led us to investigate the cellular targets of the virus in the testicles 
of newborn piglets affected by CT. By the development of an RNA in situ hybridization assay and the use of immunohistochemistry on consecutive slides, we identified the target cells of APPV in the testicle: interstitial Leydig cells, peritubular myoid cells and endothelial cells of medium-sized arteries. Previous studies have shown that APPV can be found in the semen of sexually mature boars suggesting the role of infected boars and their semen in the transmission of the virus similar to many other members of theFlaviviridae family. As in our case, the virus has not been identified in cells beyond the blood-testis barrier, further studies on infected adult boars' testicles are needed to analyze the possible changes in the cell tropism that enable the virus to be excreted by the semen.

Keywords : Pestivirus K, congenital tremor, viral tropism, immunohistochemistry, RNA ISH

\section{Introduction}

Atypical porcine pestivirus (APPV) (species Pestivirus $K$ ) belongs to the genus Pestivirus within the family of Flaviviridaeand includes highly variable RNA viruses capable of infecting cloven-hoofed animals as well as bats (Wu et al., 2012) and rats (Firth et al., 2014). They are often characterized by their detrimental effects on the embryo or the fetus, causing neuronal degeneration and subsequent neurological dysfunction and developmental anomalies such as cerebellar hypoplasia, demyelination or even stillbirth. The neurological dysfunction may result in congenital tremor (CT), also known as myoclonia congenita, which is characterized by tremors of the head and limbs of newborn piglets (Done et al., 1986). The Pestivirusgenus comprises eleven species: Pestivirus $A$ (bovine viral diarrhea virus 1), Pestivirus $B$ (bovine viral diarrhea virus 2),Pestivirus $C$ (classical swine fever virus), Pestivirus $D$ (border disease virus), Pestivirus $E$ (pronghorn pestivirus),Pestivirus $F$ (Bungowannah virus), Pestivirus $G$ (giraffe pestivirus), Pestivirus $H$ (Hobi-like pestivirus), Pestivirus I (Aydin-like pestivirus), Pestivirus $J$ (rat pestivirus) and Pestivirus $K$ (atypical porcine pestivirus) (Smith et al., 2017).

APPV was discovered in the USA in 2015, by metagenomic sequencing of a sample, which was qRT-PCR positive for porcine reproductive and respiratory syndrome virus (PRRSV) (Hause et al., 2015). To date, APPV has been identified in Germany (Postel et al., 2016; Beer et al., 2017), Sweden (Blomström et al., 2016), the Netherlands (de Groof et al., 2016), Austria (Schwarz et al., 2017), Spain (Muñoz-González et al., 2017), China (Yuan et al., 2017), Great Britain, Italy (Postel et al., 2017), Brazil (Gatto, Harmon et al., 2018), Hungary (Dénes et al., 2018), Canada (F. Dessureault et al., 2018), Switzerland (Kaufmann et al., 2019), Japan (Kasahara-kamiie et al., 2021) and most recently in Denmark (Pedersen et al., 2021). APPV was also detected in wild boars in Germany and Serbia (Cagatay et al., 2018), suggesting that, similarly to classical swine fever, wild boars may serve as a virus reservoir (Vilček and Nettleton, 2006).

Congenital tremor (CT) is characterized by tremors of the head and limbs, and it is often complicated with splayed legs (Arruda et al., 2016; de Groof et al., 2016; Schwarz et al., 2017). It has been historically classified according to the absence (type B) or presence of lesions in the central nervous system and knowledge of the etiology (type A) (Done et al., 1986; Dall Agnol et al., 2020). Type A has been divided into different subtypes from I-V, based on the causal factor (Done et al., 1986). CT caused by APPV has been associated with hypomyelination in the cerebellum and spinal cord (Schwarz et al., 2017; F. F. G. Dessureault et al., 2018; Mósena et al., 2018). Experimental studies were carried out to prove the connection between type AII CT and APPV infection and to fulfill Koch's postulates. In an experiment carried out by de Groof et al. (2017) CT was successfully induced in piglets of pregnant gilts that were inoculated intramuscularly with APPV-positive serum at 28 days of gestation (de Groof et al., 2016). CT was successfully reproduced in another experiment as well, where pregnant sows were inoculated by intravenous and intranasal routes simultaneously with direct inoculation of fetal amniotic vesicles at either 45 or 62 days of gestation. The prevalence of CT and splay leg among piglets of APPV-infected litters ranged from $57 \%$ to $100 \%$ and from $0 \%$ to $40 \%$ (Arruda et al., 2016).

Severe CT outbreaks were recorded in Austria in 2013 and 4 years later APPV was proven to be the causative agent. Malnutrition was observed in numerous piglets, overall piglet mortality was increased and the number of weaned piglets per sow decreased by 10\% (Schwarz et al., 2017). 
Other viral agents have been also proposed as possible causative agents of type AII CT. First, astroviruses and porcine circovirus type 2 (PCV2) seemed to be potential candidates, but later studies could not support these theories (Gustafson and Kaintz, 1974; Stevenson et al., 2001; Blomström et al., 2014). In a recent challenge study, pregnant gilts were inoculated with BVDV-2 but the piglets showed no signs of CT, they were PCR-negative for the presence of BVDV-2 in their serum and organ samples and the characteristic histomorphological lesions of type AII CT were absent in the CNS (Mechler et al., 2018). Linda virus, another novel porcine pestivirus was described in Austria (Lamp et al., 2017) and an experimental challenge study performed with the virus proved it to be another possible viral agent for CT (Kiesler et al., 2019).

In addition to the CNS, APPV has been detected in various other tissues such as lymphoid organs (thymus, lymph node, tonsil and spleen), digestive tract (small and large intestines, pancreas and liver), lungs and heart (Arruda et al., 2016; Postel et al., 2016). The presence of APPV in preputial fluids and semen of boars has been confirmed (de Groof et al., 2016; Schwarz et al., 2017; Gatto, Arruda et al., 2018) suggesting a potential role of insemination in virus transmission. In this sense, the male reproductive tract might serve as a virus reservoir, which has already been described for other pestiviruses such as classical swine fever virus (CSFV) (Choi and Chae, 2002) and bovine viral diarrhea virus (BVDV) (Voges et al., 1998) contributing to the persistence and transmission of the virus.

The porcine male reproductive tract is known for being highly susceptible to numerous viral infections (Phillips et al., 1972; Maes et al., 2016). The mammalian testis consists of two main compartments: (i) the interstitium, which includes the interstitial endocrine cells (Leydig cells) responsible for testosterone production, macrophages, fibroblasts and blood vessels and (ii) the seminiferous tubules, that are bordered by the peritubular myoid cells and composed of germ cells in different stages that are associated with the somatic Sertoli cells. Viruses have been found in both compartments in humans and other mammals as well (Dejucq and Jegou, 2001). The first line of testicular defence against viruses arriving from the bloodstream is the Leydig cells and testicular macrophages, whereas the second line involves the myoid cells and the Sertoli cells (Dejucq and Jegou, 2001).

As the presence of APPV in the testicles of naturally infected, CT-affected newborn piglets has not been reported so far, thus our research aimed to (i) identify the presence of the virus in the testicle by molecular methods; (ii) visualize the viral RNA by in situhybridization (RNAscope), (iii) identify the infected cell type(s) in the testis of newborn piglets by the use of immunohistochemistry on consecutive slides.

\section{Materials and Methods}

\subsection{Samples}

Processing fluid samples (the serosanguinous fluid recovered from the testicles after castration) were collected from 13 Hungarian large scale pig farms in 2018-2020 originally for the purpose of PRRSV screening (Vilalta et al., 2017; Lopez et al., 2018). The samples were kept at -80 and further analyzed for the presence of APPV.

Six 1-day-old intact male piglets from 2 different farms (3 from Farm 1, 3 from Farm 2) were humanely euthanized according to the current Hungarian animal welfare regulations (Ethical permission number: $\mathrm{PE} / \mathrm{EA} / 544-5 / 2018$ ). Two animals from each farm were affected by CT, whereas two other clinically healthy piglets served as controls. Tissue samples (cerebellum, brainstem, cerebrum, tonsil, kidney, tracheobronchial lymph node, spleen, thymus, colon and mesenteric lymph node) were collected for PCR analysis and one of the testicles were fixed in $10 \%$ neutral buffered formalin for 24 hours, then after dehydration embedded in paraffin.

\subsection{RNA extraction and $q P C R$}

The fluid fraction was collected from the bags containing the testicles as described earlier (Vilalta et al., 2017; Lopez et al., 2018), whereas the organ samples collected during the necropsies were homogenized by the use of Tissuelyzer II (Qiagen, Hilden, Germany) and centrifuged at $3000 \mathrm{rpm}$ for 3 minutes (min). RNA was extracted from the processing fluid and the tissue homogenate supernatants by Indispin/Cador Pathogen 
Mini Kit (Qiagen) according to the manufacturer's instructions in a QIAcube instrument (Qiagen). RNA was eluted in $60 \mu \mathrm{L}$ RNase free distilled water (Qiagen) and directly used for qRT-PCR analysis or archived at -80 for further analysis.

qRT-PCR analysis was implemented by QuantiNova SYBR RT-PCR Kit (Qiagen) according to the manufacturer's recommendations using primers previously designed by Kaufmann et al. (2019 for the UTR 5' region of APPV. We added $2 \mu \mathrm{L}$ template RNA to $10 \mu \mathrm{L} 2 \times$ QuantiNova SYBR Green RT-PCR Master Mix, $0.4 \mu \mathrm{M}$ of the forward and reverse primer, $0.2 \mu \mathrm{L}$ QN SYBR Green RT-Mix in a final volume of 25 $\mu \mathrm{L}$. Measurements and analysis were performed in a Rotor-Gene Q instrument (Qiagen) using the following thermal profile: 50 for $5 \mathrm{~min}, 95$ for 20 seconds (s), and 45 cycles of 95 for $3 \mathrm{~s}$, followed by 60 for $30 \mathrm{~s}$. The mean Cq values were compared by one-way ANOVAs with Tukey-Kramer post hoc tests using GraphPad Prism version 8.0.1 for Windows ("GraphPad Software," n.d.) and the statistical significance was set at $\mathrm{P}<0.05$.

\section{3. $A P P V$ in situ hybridization (RNAscope)}

RNA in situ hybridization (ISH) for the APPV genome in the testicles was performed manually using RNAscope@ 2.5 HD Detection Kit (RED) (Advanced Cell Diagnostics, Newark, CA, USA) according to the manufacturer's instructions using probes that were designed to bind to the sequence region of nucleotides 1-2816 of the genes encoding the NS2-NS3 proteins. Briefly, $5 \mu \mathrm{m}$ thin sections were cut from formalinfixed, paraffin-embedded (FFPE) blocks and were pretreated with heat and protease before hybridization with the target oligonucleotide probes. Preamplifier, amplifier, and AP-labeled oligos were then hybridized sequentially, followed by chromogenic precipitate development. The integrity of the RNA and the specificity of the reaction was tested with a positive control probe specific to Ss-PPIB RNA (Sus scrofa peptidylprolyl isomerase B) and with a negative control probe specific to bacterial dapB RNA (Bacillus subtilisdihydrodipicolinate reductase), respectively. Testicles obtained from the healthy piglets served as virus negative control.

\subsection{Immunohistochemistry}

The immunohistochemical (IHC) antibodies for the identification of different cell types were anti-von Willebrand Factor (vWF, polyclonal rabbit anti-human, diluted 1:500, DAKO, Glostrup, Denmark) for the endothelial cells; anti-c-kit (diluted 1:200, polyclonal rabbit anti-human, DAKO) for the Leydig cells and germ cells, anti-alpha smooth muscle actin ( $\alpha$-SMA, diluted 1:100, monoclonal mouse anti-human clone1A4, DAKO) for the detection of contractile smooth muscle elements in various cell types.

For the IHC investigations, consecutive 3-4 $\mu \mathrm{m}$ thin sections were cut from the FFPE blocks of the testicles. The slides were deparaffinized in xylene $(2 \times 10 \mathrm{~min})$ and $96 \%$ alcohol $(2 \times 5 \mathrm{~min})$. The deparaffinized sections were treated with peroxidase-blocking reagent (EnVision FLEX, Agilent, Santa Clara, CA, USA) for 5 min after treatment with appropriate target retrieval (Target Retrieval Solution, DAKO) $\mathrm{pH}=9$, in a microwave oven at $800 \mathrm{~W}$, for $25 \mathrm{~min}$ in case of the vWF and c-kit, and $10 \mathrm{~min}$ in the case of $\alpha$-SMA. Sections were treated with the given primary antibody for $30 \mathrm{~min}$ at room temperature. Antigen-bound primary antibody was detected using standard avidin-biotin immunoperoxidase complex (EnVision FLEX HRP kit, Agilent), using 3, 3 - diaminobenzidine tetrahydrochloride (DAB substrate- chromogen, DAKO) as chromogen substrate. Counterstaining was performed with Hematoxylin GILL II (Merck, Darmstadt, Germany) for $30 \mathrm{~s}$. Finally, before mounting, the sections were treated with graded ethanol $(2 \times 5 \mathrm{~min})$ and xylene $(2 \times 5 \mathrm{~min})$. For both ISH and IHC, reactions were carried out parallelly on the testicles of every six animals.

Both ISH and IHC treated slides were scanned with Pannoramic Midi slide scanner instrument (3DHistech, Budapest, Hungary), the digital slides were analyzed, and the representative pictures were taken with the CaseViewer software (3DHistech). The MultiView Toolbox function of the Case Viewer software was used to align the digital version of the consecutive slides to each other.

\section{Results}




\subsection{Quantitative real-time PCR}

We detected APPV in 10 out of the 13 investigated farms in the processing fluid samples. Only 2 of these farms have ever experienced CT among newborn piglets. APPV was also detected in the testicles of all four CT-affected animals by qRT-PCR (and RNAscope), while the testicles of unaffected animals showed no viral presence (Table 1).

By qRT-PCR assay every organ sample collected during the necropsies (cerebellum, brainstem, cerebrum, tonsil, kidney, tracheobronchial lymph node, spleen, thymus, colon, and mesenteric lymph node samples) of the CT-affected animals showed positivity (except the mesenteric lymph node of Animal No. V.) and, interestingly, APPV was also detected in the cerebellum $(\mathrm{Cq}$ value 30.14$)$ and the tonsil (Cq value 26.53) of the clinically healthy Animal No. I.

There was no significant difference between the mean Cq values of tissue types (Figure 1).

\subsection{RNA in situ hybridization and immunohistochemistry}

RNA integrity and tissue fixation/pretreatment conditions proved to be adequate as no red signal was detected in the slides treated with the negative control probe, (Figure 2/A), while an intense red signal was apparent in the case of the housekeeping positive control probe (Figure 2/B).

APPV infected cells in the testicular slides of CT-affected piglets were successfully identified by RNAscope in all four cases (II, III, V, VI) as intracytoplasmatic red signal. The positive cells were found in three distinct compartments of the testis: among cells of the otherwise prominent testicular interstitium, in fusiform cells around the primitive, non-functional seminiferous tubules and also in some of the medium-sized (noncapillary) arteries (Figure 3).

For the identification of these cells, we used multiple immunohistochemistry stainings on consecutive slides. Endothelial cells of the blood vessels were labelled with anti-vWF antibody (Figure 4/B), interstitial Leydig cells and germ cells were identified by anti-c-kit antibody (Figure 4/C) and smooth muscle containing cells around the seminiferous tubules were labelled with anti- $\alpha$-SMA antibody (Figure $3 / \mathrm{A}$ ), whereas the same area stained by RNAscope ISH is also shown in Figure 4/D.

In some areas, the distribution of vWF positive cells was similar to the ones labelled with $\alpha$-SMA due to the double positivity of the blood vessels. However, intense cytoplasmic positivity of the peritubular fusiform cells was only observed in the case of $\alpha$-SMA labelling, indicating the presence of smooth muscle fibers in their cytoplasm without being related to blood vessels. These cells are the peritubular myoid cells in the testicle. According to the results obtained by the different immunohistochemical and RNAscope staining of the consecutive slides suggests that APPV is predominantly localized in the Leydig cells of the interstitium as well as in the peritubular myoid cells located around the seminiferous tubules (Figure 3 A-D). No viral genome was detected in the germ cells and the Sertoli cells as well as in the negative control animals, and similar distribution of the ISH positivity was observed in the testicles of every CT-affected animal.

\section{Discussion}

During the screening of testicular processing fluid samples obtained for PRRSV monitoring of newborns, we found unexpectedly high prevalence of APPV in this sample type even in farms that never experienced CT outbreaks. Processing fluid is primarily used for PRRSV screening in newborn animals, as the presence of the virus in this age group refers to active virus circulation in the breeding herd (Vilalta et al., 2017; Lopez et al., 2018). To our knowledge this is the first report on the use of processing fluid to detect APPV in piglets. The high rate of detection suggests widespread congenital infection of the newborn piglets even in the absence of CT. This finding and the testicular tropism of other members of the Pestivirus genus led us to further investigate the organs of naturally infected newborn piglets with special emphasis on the testicles.

We obtained 2-2 CT-affected newborn male piglets and 1-1 control animals from large scale swine farms and collected organ samples to verify the presence of APPV in them. Real-time qPCR analysis revealed APPV 
positivity in every tested organ of the CT-affected animals with no significant differences in the viral loads. The healthy age-matched control animals were negative for the presence of the virus.

There are only a few reports in the literature investigating the target cells in the testis in case of a pestivirus infection (Voges et al., 1998; Choi and Chae, 2002; Read et al., 2020; Storino et al., 2020). A closely related pestivirus, the BVDV was identified in the testes of a post-puberty bull, persistently shedding the virus in the semen for almost a year. The authors found that the virus was sequestered in the testes, therefore it was hypothesized, that the bull was infected after puberty, as the virus was protected in the testes from the bull's immune response by the blood-testes barrier, which only becomes functional after puberty when the tight junctions between the Sertoli cells are formed (Voges et al., 1998).

More recent research analyzed the testicles of bulls, that had persistent BVDV infection confined to their reproductive tract shedding the virus through semen for a prolonged period. The authors showed that the virus was localized in the seminiferous tubules, resulting in a reduced or collapsed lumen diameter lined by a Sertoli cell layer or near-total loss of Sertoli cells, leading to the increased prominence of Leydig cells. Furthermore, BVDV was also detected in still-existing germinal cell stages (Read et al., 2020). Another study concerning BVDV showed no virus shedding through the reproductive route, although the duration of the study was shorter (Storino et al., 2020). On the other hand, classical swine fever virus, another closely related pestivirus of pigs was not detected in Sertoli, Leydig or endothelial cells of the testicle, but it was identified in monocytes and macrophages with large oval nuclei and abundant cytoplasm (Choi and Chae, 2002).

In our study APPV genome was successfully identified by the RNA based ISH method (RNAscope) in the interstitial Leydig cells, in endothelial cells of medium-sized arteries and spindle-shaped mesenchymal cells around the seminiferous tubules. On consecutive sections, the latter cells showed intense cytoplasmatic positivity for $\alpha$-SMA and were negative for vWF suggesting that they are smooth muscle fiber bearing cells that are not related to blood vessels. These cells can be identified as the peritubular myoid cells of the testicle. Interestingly, we could not identify positive signal in the endothelial cells of capillaries even though the infection of these cells has been already confirmed for other organs in previous studies (Liu et al., 2019)(Postel et al., 2016; Hu et al., 2019; Liu et al., 2019). Peritubular myoid cells are smooth muscle cells surrounding the seminiferous tubules. They provide the tubular structural integrity, play a role in the regulation of spermatogenesis and with their muscle fibers they take part in the contractions of the seminiferous tubule to aid the transport of spermatozoa in the tubular lumen (Maekawa et al., 1996). Leydig cells are also located in the intertubular compartment and are responsible for testosterone hormone production which is important to maintain spermatogenesis and the male secondary sex characteristics (Mita et al., 2011).

In our study testicles of newborn animals were tested, in which the relative volume of the intertubular compartment - predominantly composed of Leydig cells and blood vessels - is significantly higher than in adults and is gradually decreasing till sexual maturity. As expected, an opposite trend can be observed for the volume of the tubular compartment (Avelar et al., 2010).

Schwarz et al. (2017) followed the course of viraemia of CT affected piglets for 6 months and found high levels of APPV genome $\left(2.1 \times 10^{9} \mathrm{GE} / \mathrm{mL}\right)$ in the semen of a boar that reached sexual maturity by that time. Even though the authors did not perform further investigations on their reproductive organs, for such a prolonged infection the virus must establish persistent infection in the seminiferous tubules, which is an immune-privileged site in the testis protected by the blood-testis barrier. This phenomenon has been observed in bulls with prolonged testicular infection of BVDV (Read et al. 2020) and also in the case of Zika virus another flavivirus of human beings (Siemann et al., 2017), both of which are known for being transmitted via semen. In our case, the viral genome was not observed in cells beyond the blood-testis barrier (germ cells or Sertoli cells).

Based on our results and the study by Schwarz et al. (2017) it can be speculated that APPV infects different cells in the testicles of newborns compared to adult animals and in the case of prolonged testicular APPV 
infection the virus could be able to gain access to cells beyond the blood-testis barrier to infect Sertoli cells and germ cells, enabling the virus to be excreted into the semen, as it has already been described for BVDV in bulls (Read et al., 2020). It can not be excluded though that other cells of the male reproductive glands are infected as well that also release their content along with APPV in the semen. The latter cells however are not that well protected from the immune system of the animal.

An important observation of our study is that piglets born in APPV infected farms with or without CT can be a source of infection for other age groups, and the testicles should be collected and discarded after castration preventing sows or other piglets to get in contact with them. Moreover, piglets that are born with symptoms of CT - even though they might recover from shaking and become clinically healthy - should not be kept for breeding purposes as they might be shedding the virus in their semen for a longer period. We have also proved that processing fluid is a cheap and useful diagnostic sample for the detection of APPV in a given farm, that does not require additional stressful/painful handling of the animals.

Out future aim is to screen boar studs in our country for the presence of APPV in semen, estimate the prevalence of the infection/virus excretion, identify positive animals and in the case of culling, we will perform the same investigations on their testicles. Those results can reveal the actual role of insemination and semen transport in the transmission and epidemiology of APPV and identify the cellular sources of virus shedding in the semen.

To the best of our knowledge, our study is the first to describe the development and practical use of a chromogenic RNA based in situhybridization assay to analyze the cellular targets of APPV. Consecutive slides prepared from the testicles of newborn male piglets and labelled with anti-vWF, anti-c-kit, anti- $\alpha \mathrm{SMA}$ antibodies by IHC and RNAscope ISH showed that the virus was localized in the intertubular compartment and in the peritubular myoid cells of the testis without the involvement of the germ cells and Sertoli cells beyond the blood-testis barrier.

\section{Acknowledgements}

Project no. TKP2020-NKA-01 has been implemented with the support provided from the National Research, Development and Innovation Fund of Hungary, financed under the Tématerületi Kiválósági Program 2020 (2020-4.1.1-TKP2020) funding scheme. Gyula Balka was supported by the János Bolyai Research Scholarship of the Hungarian Academy of Sciences and by the Bolyai + Fellowship (ÚNKP-20-5-ÁTE-2) of the New National Excellence Program of the Hungarian Ministry of Innovation and Technology. Lilla Dénes was supported by the ÚNKP-4 New National Excellence Program of the Ministry of Human Capacities. The funding sources had no role in study design, data collection and analysis or the decision to publish the work.

\section{Conflict of Interest}

The authors declare no conflict of interest.

\section{References}

Arruda, B.L., P.H. Arruda, D.R. Magstadt, K.J. Schwartz, T. Dohlman, J.A. Schleining, A.R. Patterson, C.A. Visek, and J.G. Victoria, 2016: Identification of a Divergent Lineage Porcine Pestivirus in Nursing Piglets with Congenital Tremors and Reproduction of Disease following Experimental Inoculation. (Frederick C. Leung, Ed.)PLoS One11, e0150104, DOI: 10.1371/journal.pone.0150104.

Avelar, G.F., C.F.A. Oliveira, J.M. Soares, I.J. Silva, I. Dobrinski, R.A. Hess, and L.R. França, 2010: Postnatal somatic cell proliferation and seminiferous tubule maturation in pigs: A non-random event. Theriogenology 74 , 11-23, DOI: 10.1016/j.theriogenology.2009.12.014.

Beer, M., K. Wernike, C. Dräger, D. Höper, A. Pohlmann, C. Bergermann, C. Schröder, S. Klinkhammer, S. Blome, and B. Hoffmann, 2017: High Prevalence of Highly Variable Atypical Porcine Pestiviruses Found in Germany. Transbound. Emerg. Dis. 64, e22-e26, DOI: 10.1111/tbed.12532.

Blomström, A.L., C. Fossum, P. Wallgren, and M. Berg, 2016: Viral metagenomic analysis displays the 
Co-infection situation in healthy and PMWS affected pigs. PLoS One 11, 1-11, DOI: 10.1371/journal.pone.0166863.

Blomström, A.L., C. Ley, and M. Jacobson, 2014: Astrovirus as a possible cause of congenital tremor type AII in piglets? Acta Vet. Scand.56 , 82, DOI: 10.1186/s13028-014-0082-y.

Cagatay, G.N., A. Antos, D. Meyer, C. Maistrelli, O. Keuling, P. Becher, and A. Postel, 2018: Frequent infection of wild boar with atypical porcine pestivirus (APPV). Transbound. Emerg. Dis. 65 , 1087-1093, DOI: $10.1111 /$ tbed.12854.

Choi, C., and C. Chae, 2002: Localization of classical swine fever virus in male gonads during subclinical infection. J. Gen. Virol.83, 2717-2721, DOI: 10.1099/0022-1317-83-11-2717.

Dall Agnol, A.M., A.F. Alfieri, and A.A. Alfieri, 2020: Pestivirus K (Atypical Porcine Pestivirus): Update on the Virus, Viral Infection, and the Association with Congenital Tremor in Newborn Piglets. Viruses 12 , 903, DOI: $10.3390 / \mathrm{v} 12080903$.

de Groof, A., M. Deijs, L. Guelen, L. van Grinsven, L. van Os-Galdos, W. Vogels, C. Derks, T. Cruijsen, V. Geurts, M. Vrijenhoek, J. Suijskens, P. van Doorn, L. van Leengoed, C. Schrier, and L. Hoek, 2016: Atypical Porcine Pestivirus: A Possible Cause of Congenital Tremor Type A-II in Newborn Piglets. Viruses 8, 271, DOI: $10.3390 / \mathrm{v} 8100271$.

Dejucq, N., and B. Jegou, 2001: Viruses in the Mammalian Male Genital Tract and Their Effects on the Reproductive System. Microbiol. Mol. Biol. Rev. 65 , 208-231, DOI: 10.1128/mmbr.65.2.208-231.2001.

Denes, L., I. Biksi, M. Albert, L. Szeredi, D.G. Knapp, A. Szilasi, A. Balint, and G. Balka, 2018: Detection and phylogenetic characterization of atypical porcine pestivirus strains in Hungary. Transbound. Emerg. Dis. 65 , 2039-2042, DOI: 10.1111/tbed.12981.

Dessureault, F., C. M, P. C, and G. CA., 2018: First report of atypical porcine pestivirus in piglets with congenital tremor in Canada.Can Vet J. 59, 429-432.

Dessureault, F.F.G., C. M, P. C, G. CA., M. Choiniere, C. Provost, and C.A. Gagnon, 2018: First report of atypical porcine pestivirus in piglets with congenital tremor in Canada. Can. Vet. J. = La Rev. Vet. Can. $\mathbf{5 9}, 429-432$.

Done, J.T., J. Woolley, D.H. Upcott, and C.N. Hebert, 1986: Porcine congenital tremor type AII: Spinal cord morphometry. Br. Vet. J.142 , 145-150, DOI: 10.1016/0007-1935(86)90090-4.

Firth, C., M. Bhat, M.A. Firth, S.H. Williams, M.J. Frye, P. Simmonds, J.M. Conte, J. Ng, J. Garcia, N.P. Bhuva, B. Lee, X. Che, P.L. Quan, and W. Ian Lipkin, 2014: Detection of zoonotic pathogens and characterization of novel viruses carried by commensal rattus norvegicus in New York city. MBio $\mathbf{5}, 1-16$, DOI: $10.1128 / \mathrm{mBio} .01933-14$.

Gatto, I.R.H., P.H. Arruda, C.A. Visek, J.G. Victoria, A.R. Patterson, A.C. Krull, K.J. Schwartz, L.G. de Oliveira, and B.L. Arruda, 2018: Detection of atypical porcine pestivirus in semen from commercial boar studs in the United States. Transbound. Emerg. Dis. 65, e339-e343, DOI: 10.1111/tbed.12759.

Gatto, I.R.H., K. Harmon, L. Bradner, P. Silva, D.C.L. Linhares, P.H. Arruda, L.G. de Oliveira, and B.L. Arruda, 2018: Detection of atypical porcine pestivirus in Brazil in the central nervous system of suckling piglets with congenital tremor. Transbound. Emerg. Dis.65 , 375-380, DOI: 10.1111/tbed.12824.

Gustafson, D.P., and C.L. Kaintz, 1974: Experimental transmission of congenital tremors in swine. Proc. Annu. Meet. U. S. Anim. Health Assoc. 338-45.

Hause, B.M., E.A. Collin, L. Peddireddi, F. Yuan, Z. Chen, R.A. Hesse, P.C. Gauger, T. Clement, Y. Fang, and G. Anderson, 2015: Discovery of a novel putative atypical porcine pestivirus in pigs in the USA. J. Gen. Virol. 96 , 2994-8, DOI: 10.1099/jgv.0.000251. 
Hu, H.Z.W., J.L.T. Liu, and T.O.C. Xiao, 2019: Novel circovirus species identified in farmed pigs designated as Porcine circovirus 4, Hunan province, China. 1-5, DOI: 10.1111/tbed.13446.

Kasahara-kamiie, M., M. Kagawa, M. Shiokawa, F. Sunaga, N. Aihara, T. Shiga, J. Kamiie, and H. Aoki, 2021: Detection and genetic analysis of a novel atypical porcine pestivirus from piglets with congenital tremor in Japan. 1-10.

Kaufmann, C., H. Stalder, X. Sidler, S. Renzullo, C. Gurtner, A. Grahofer, S. Schweizer, and M. Schweizer, 2019: Long-term circulation of atypical porcine pestivirus (APPV) within switzerland. Viruses11, 1-14, DOI: $10.3390 / \mathrm{v} 11070653$.

Kiesler, A., K. Seitz, L. Schwarz, K. Buczolich, H. Petznek, E. Sassu, S. Durlinger, S. Hogler, A. Klang, C. Riedel, H.-W.W. Chen, M. Motz, P. Kirkland, H. Weissenbock, A. Ladinig, T. Rumenapf, and B. Lamp, 2019: Clinical and Serological Evaluation of LINDA Virus Infections in Post-Weaning Piglets. Viruses 11, 975, DOI: $10.3390 / \mathrm{v} 11110975$.

Lamp, B., L. Schwarz, S. Hogler, C. Riedel, L. Sinn, B. Rebel-Bauder, H. Weissenbock, A. Ladinig, and T. Rumenapf, 2017: Novel pestivirus species in pigs, Austria, 2015. Emerg. Infect. Dis. 23, 1176-1179, DOI: 10.3201/eid2307.170163.

Liu, J., Z. Li, X. Ren, H. Li, R. Lu, Y. Zhang, and Z. Ning, 2019: Viral load and histological distribution of atypical porcine pestivirus in different tissues of naturally infected piglets. Arch. Virol.164, 2519-2523, DOI: $10.1007 / \mathrm{s} 00705-019-04345-3$.

Lopez, W., J. Angulo, and J. Zimmerman, 2018: Porcine reproductive and respiratory syndrome monitoring in breeding herds using processing fluids. J. Swine Heal. Prod. 26 , 146-150.

Maekawa, M., K. Kamimura, and T. Nagano, 1996: Peritubular myoid cells in the testis: Their structure and function. Arch. Histol. Cytol. 59, 1-13, DOI: 10.1679/aohc.59.1.

Maes, D., A. Van Soom, R. Appeltant, I. Arsenakis, and H. Nauwynck, 2016: Porcine semen as a vector for transmission of viral pathogens. Theriogenology 85 ,27-38, DOI: 10.1016/j.theriogenology.2015.09.046.

Mechler, M.L., F. dos S. Gomes, K.A. Nascimento, A. de Souza-Pollo, F.F.B. Pires, S.I. Samara, E.M. Pituco, and L.G. de Oliveira, 2018: Congenital tremor in piglets: Is bovine viral diarrhea virus an etiological cause? Vet. Microbiol. 220 , 107-112, DOI: 10.1016/j.vetmic.2018.05.009.

Mita, P., B.T. Hinton, and J.M. Dufour, 2011: The blood-testis and blood-epididymis barriers are more than just their tight junctions. Biol. Reprod. 84, 851-858, DOI: 10.1095/biolreprod.110.087452.

Mosena, A.C.S., M.N. Weber, R.A.S. da Cruz, S.P. Cibulski, M.S. da Silva, D.E. Puhl, M.E. Hammerschmitt, K.L. Takeuti, D. Driemeier, D.E.S.N. de Barcellos, and C.W. Canal, 2018: Presence of atypical porcine pestivirus (APPV) in Brazilian pigs. Transbound. Emerg. Dis. 65 , 22-26, DOI: 10.1111/tbed.12753.

Munoz-Gonzalez, S., A. Canturri, M. Perez-Simo, J.A. Bohorquez, R. Rosell, O. Cabezon, J. Segales, M. Domingo, and L. Ganges, 2017: First report of the novel atypical porcine pestivirus in Spain and a retrospective study. Transbound. Emerg. Dis. 64, 1645-1649, DOI: 10.1111/tbed.12699.

Pedersen, K., C.S. Kristensen, B. Strandbygaard, A. Botner, and T.B. Rasmussen, 2021: Detection of atypical porcine pestivirus in piglets from Danish sow herds. Viruses 13 , 1-7, DOI: 10.3390/v13050717.

Phillips, R., C. Foley, and P. Lukert, 1972: ISOLATION AND CHARACTERIZATION OF VIRUSES FROM SEMEN AND THE REPRODUCTIVE TRACT OF MALE SWINE. J. Am. Vet. Med. Assoc. 161 , 1306-1316.

Postel, A., F. Hansmann, C. Baechlein, N. Fischer, M. Alawi, A. Grundhoff, S. Derking, J. Tenhundfeld, V.M. Pfankuche, V. Herder, W. Baumgartner, M. Wendt, and P. Becher, 2016: Presence of atypical porcine pestivirus (APPV) genomes in newborn piglets correlates with congenital tremor. Sci. Rep. 6, 27735, DOI: $10.1038 /$ srep27735. 
Postel, A., D. Meyer, G.N. Cagatay, F. Feliziani, G.M. De Mia, N. Fischer, A. Grundhoff, V. Milićević, M.-C. Deng, C.-Y. Chang, H.-J. Qiu, Y. Sun, M. Wendt, and P. Becher, 2017: High Abundance and Genetic Variability of Atypical Porcine Pestivirus in Pigs from Europe and Asia.Emerg. Infect. Dis. 23 , 2104-2107, DOI: 10.3201/eid2312.170951.

Read, A.J., S. Gestier, K. Parrish, D.S. Finlaison, X. Gu, T.W. O'Connor, and P.D. Kirkland, 2020: Prolonged detection of bovine viral diarrhoea virus infection in the semen of bulls. Viruses12, DOI: $10.3390 / \mathrm{v} 12060674$.

Schwarz, L., C. Riedel, S. Högler, L.J. Sinn, T. Voglmayr, B. Wöchtl, N. Dinhopl, B. Rebel-Bauder, H. Weissenböck, A. Ladinig, T. Rümenapf, and B. Lamp, 2017: Congenital infection with atypical porcine pestivirus (APPV) is associated with disease and viral persistence. Vet. Res. 48, 1, DOI: 10.1186/s13567016-0406-1.

Siemann, D.N., D.P. Strange, P.N. Maharaj, P.-Y. Shi, and S. Verma, 2017: Zika Virus Infects Human Sertoli Cells and Modulates the Integrity of the In Vitro Blood-Testis Barrier Model. (Adolfo García-Sastre, Ed.) J. Virol. 91 , 1570-1580, DOI: 10.1128/JVI.00623-17.

Smith, D.B., G. Meyers, J. Bukh, E.A. Gould, T. Monath, A. Scott Muerhoff, A. Pletnev, R. Rico-Hesse, J.T. Stapleton, P. Simmonds, P. Becher, A.S. Muerhoff, A. Pletnev, R. Rico-Hesse, J.T. Stapleton, P. Simmonds, and P. Becher, 2017: Proposed revision to the taxonomy of the genus Pestivirus, family Flaviviridae. J. Gen. Virol.98 , 2106-2112, DOI: 10.1099/jgv.0.000873.

Stevenson, G.W., M. Kiupel, S.K. Mittal, J. Choi, K.S. Latimer, and C.L. Kanitz, 2001: Tissue distribution and genetic typing of porcine circoviruses in pigs with naturally occurring congenital tremors.J. Vet. Diagnostic Investig. 13 , 57-62, DOI: 10.1177/104063870101300111.

Storino, G.Y., E.B. Xavier, M.L. Mechler-dreibi, A. Simonatto, I.R.H. Gatto, M.E.F. Oliveira, E.M. Pituco, L.G. de Oliveira, G. Yuri, E. Bellini, M.L. Mechler-dreibi, A. Simonatto, I. Renan, H. Gatto, M. Emilia, F. Oliveira, E. Maristela, L. Guilherme, and D. Oliveira, 2020: No effects of noncytopathic bovine viral diarrhea virus type 2 on the reproductive tract of experimentally inoculated boars. Vet. Microbiol. 240 , 108512, DOI: 10.1016/j.vetmic.2019.108512.

Vilalta, C., J. Sanhueza, M. Torremorell, and B. Morrison, 2017: Use of processing fluids for PRRSV diagnostics. 2017.

Vilček, Š., and P.F.F. Nettleton, 2006: Pestiviruses in wild animals. Vet. Microbiol. 116 , 1-12, DOI: 10.1016/j.vetmic.2006.06.003.

Voges, H., G.. W. Horner, S. Rowe, and G.. J. Wellenberg, 1998: Persistent bovine pestivirus infection localized in the testes of an immuno-competent, non-viraemic bull. Vet. Microbiol. 61, 165-175, DOI: 10.1016/S0378-1135(98)00177-1.

Wu, Z., X. Ren, L. Yang, Y. Hu, J. Yang, G. He, J. Zhang, J. Dong, L. Sun, J. Du, L. Liu, Y. Xue, J. Wang, F. Yang, S. Zhang, and Q. Jin, 2012: Virome Analysis for Identification of Novel Mammalian Viruses in Bat Species from Chinese Provinces. J. Virol. 86 , 10999-11012, DOI: 10.1128/JVI.01394-12.

Yuan, J., Z. Han, J. Li, Y. Huang, J. Yang, H. Ding, J. Zhang, M. Zhu, Y. Zhang, J. Liao, M. Zhao, and J. Chen, 2017: Atypical Porcine Pestivirus as a Novel Type of Pestivirus in Pigs in China. Front. Microbiol. 8, 1-6, DOI: 10.3389/fmicb.2017.00862.

N.d.: GraphPad Software [Online] Available at www.graphpad.com (accessed March 30, 2021).

Table legend

Table 1. APPV detection in the testicles of the animals included in the study by qRT-PCR and RNAscope.

Figure legends 
Figure 1. Tissue tropism of atypical porcine pestivirus (APPV). Average qRT-PCR Cq values by sample type of the four $\mathrm{CT}$ affected piglets are shown, with \pm 1 standard deviation as error bars.

Figure 2. A negative control probe (dapB, A) and a positive control probe (Ss-PPIB, B) were used for RNAscope, testing the integrity of RNA.

Figure 3. Representative pictures of APPV RNAscope ISH genome on the testicle samples. Black arrows point at positive peritubular myoid cells, red arrows show the signal in the interstitial Leydig cells, whereas the white arrows indicate positive endothelial cells in the wall of medium-sized arteries marked by asterisks. $600 \times$, Bar $=20 \mu \mathrm{m}$.

Figure 4. Representative pictures of $\alpha$-SMA (A), vWF (B), c-kit (C) IHC stainings and the RNAscope ISH (D) on consecutive slides. Black asterisks in the left bottom corner indicate the same artery, dashed circles in pictures B and D indicate capillaries in the interstitial compartment. White arrows point at $\alpha$-SMA positive peritubular myoid cells in picture A, red asterisks show the c-kit positive interstitial Leydig cells in picture C, whereas the black arrows point at APPV genome labelled as red ISH signal in picture D. $300 \times$, Bar $=$ $50 \mu \mathrm{m}$.
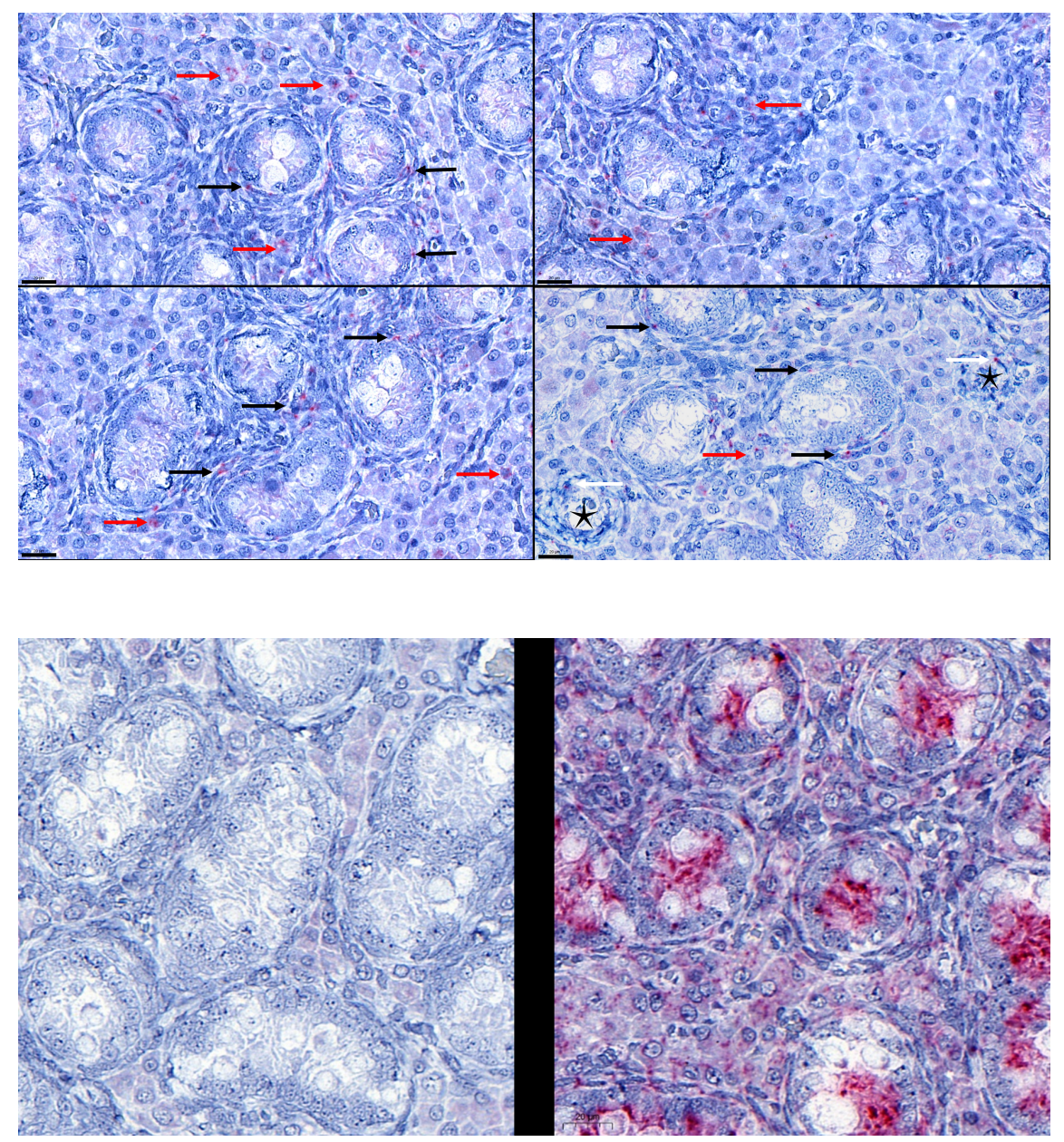

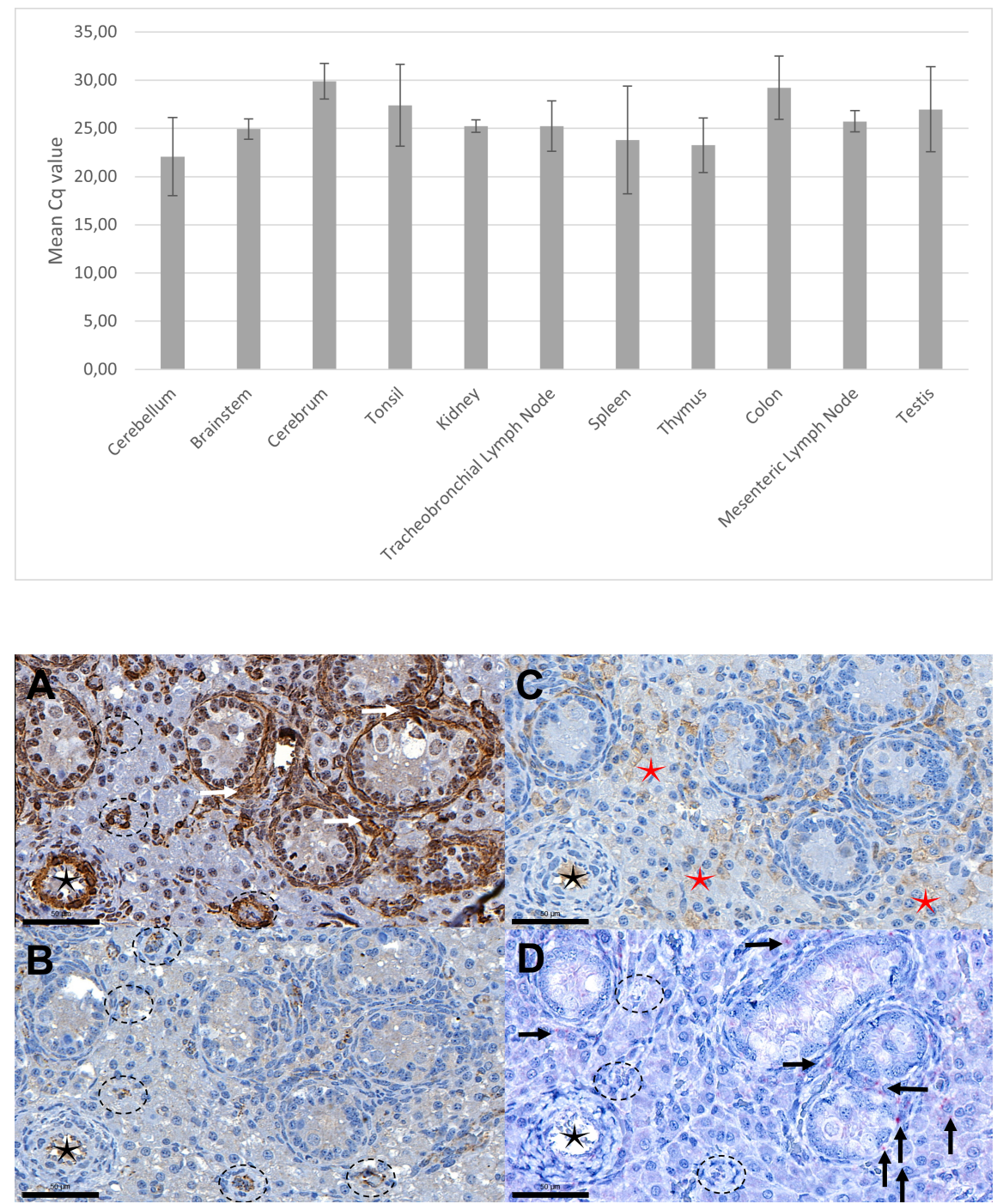

\section{Hosted file}

Table 1.docx available at https://authorea.com/users/345245/articles/531571-detection-andlocalization-of-atypical-porcine-pestivirus-in-the-testicles-of-naturally-infected-ctaffected-piglets 